\title{
Role of lymphangiogenesis in lung cancer
}

\author{
Bożena Weryńska1, Piotr Dzięgiel ${ }^{2,3}$, Renata Jankowska ${ }^{1}$ \\ ${ }^{1}$ Chair and Department of Pulmonology and Pulmonary Tumours, Medical University in Wrocław, Wrocław, \\ Poland \\ ${ }^{2}$ Department of Histology and Embryology, Medical University in Wrocław, Wrocław, Poland \\ ${ }^{3}$ Department of Histology and Embryology, University of Medical Sciences in Poznań, Poznań, Poland
}

\begin{abstract}
Lung cancer represents one of the most frequent causes of death due to neoplastic disease in Poland and around the world. The high mortality which accompany neoplastic diseases used to be ascribed mainly to dissemination of cancerous cells. Studies on animal models suggest that tumour lymphangiogenesis represents the principal factor in the process of metastases formation. Lymphangiogenesis involves a process of formation of new lymphatic vessels from already existing lymphatic capillaries. Lymphangiogenesis is stimulated by vascular endothelial growth factors (VEGF) and other, recently reported factors, such as, e.g., cyclooxygenase 2, fibroblast growth factor 2, angiopoetin-1 and the insulin-resembling growth factor. In lymphangiogenesis a key role is played by neutropilin 2 or podoplanin and this promoted development of studies on lymphangiogenesis. Activation of VEGF-C/VEGF-D/VEGFR-3 axis increases motility and invasiveness of neoplastic cells, promotes development of metastases in several types of tumours such as, e.g., lung cancer, mammary carcinoma, cancers of the neck, prostate and large intestine. In recent years lymphangiogenesis provided topic of many studies. A positive correlation was detected between expressions of VEGF-C/D and VEGFR-3 in non-small cell lun cancer. In patients with lung cancer with high expression of VEGF-C a markedly abbreviated survival was noted. Positive correlation was detected between expression of VEGF-C and VEGF-D on one hand and expression of LYVE-1 on the other in sentinel lymph nodes with metastases of neoplastic cells in patients with non-small cell lung cancer. Also, high density of lymphatic vessels and high density of intraneoplastic microvessels proved to be independent poor prognostic indices in patients with non-small cell lung cancer. Extensive hope is linked to studies on inhibitors of lymphangiogenesis, which may improve results of treatment also in tumour patients.
\end{abstract}

Key words: lung cancer, lymphangiogenesis, vascular endothelial growth factors, markers of lymphatic vessels

\section{Introduction}

Lung cancer represents the most frequent cause of death due to a neoplastic disease, the most frequent malignant tumour in the world and in Poland the most frequent malignant tumour in males [1-3]. The high mortality which accompanies neoplastic disease is ascribed mainly to the spread of cancerous cells $[4,5]$. Role of angiogenesis in development of neoplastic metastases has been relatively well recognised while the role of lymphangiogenesis in the process remains incompletely clarified $[6,7]$. Studies on animal models suggest that tumour lymphangiogenesis plays leading role in the metastatic process [8].

Correspondence: P. Dzięgiel, Zakład Histologii i Embriologii Akademii Medycznej we Wrocławiu, ul. Chałubińskiego 6a, 50-368 Wrocław, tel: +48 71/7840081, fax: +48 71/7840082, e-mail: piotr@hist.am.wroc.pl
Structure of lymphatic vessels promotes dissemination of tumour cells. They represent thin-walled, low pressure vessels, which transport intercellular fluid, proteins, lymphocytes and antigen-presenting cells to lymph nodes and to circulation. On the other hand, blood vessels work under higher internal pressures and their wall in thicker [9]. Neoplastic cells which penetrate lymphatic vessels may be passed to the circulation by the thoracic duct or by side anastomoses (leaks), probably existing in the tumour structure between blood and lymphatic systems [10].

Role of lymphangiogenesis, probably responsible also for metastases in a neoplastic disease has provided topic of few reports in the recent decade. This reflected, among other, the absence of specific markers which might specifically identify lymphatic vessels. 


\section{Structure and function of lymphatic system}

The lymphatic system was described for the first time by Gasparo Aselli in 1627. In 1902, Florence Sabin advanced a theory related to formation of lymphatic system during embryogenesis. According to the theory, peripheral lymphatic system developed from primitive lymphatic vesicles, arising due to budding of of venous endothelial cells. The report of 2002, published by Jussila and Alitalo, confirmed the theory of Sabin [11]. The lymphatic system consists of thin-walled, low pressure vessels, lymph nodes localised along the vessels, lymphoid organs such as the spleen and the thymus and of circulating lymphocytes [12]. Due to the control of intercellular fluid transport, the lymphatic system maintains an appropriate plasma volume, prevents against pressure build-up in tissues and plays a significant role in the immune defence system of the body [13].

Lymphatic vessels differ in their function and structure from those of blood vessels. Walls of lymphatic vessels are thin, cytoplasm of endothelial cells is less dense, basement membrane is discontinuous, which increases permeability of the vessels. Lumen of lymphatic vessels shows a three-fold higher diameter than lumen of small blood vessels, shape of lymphatic vessels is less regular and the vessels may be occluded (frequently collapsed) [14]. The wall of lymphatic vessels is joined to the extracellular matrix by reticular and collagen fibres. With an increasing inflow of intercellular fluid and in conditions of an increased pressure the connective tissue fibres stretch to open vascular lumen [15]. Lymph flow is facilitated by contractions of smooth muscles which surround the vessels and a direction of flow is enforced by the system of valves in lymphatic vessels [14]. Destruction or a disturbed continuity of lymphatic vessels by infectious, mechanical agents, obliteration, irradiation, or surgical procedures lead to oedema in the lymphatic system and to increased pressure in the surrounding tissues [16].

\section{Markers of lymphatic vessels}

Lymphangiogenesis involves a process of new lymphatic vessel formation from the already existing lymphatic capillaries [17]. Progress in studies on lymphangiogenesis was hampered due to the lack of specific markers of lymphatic system. In the past, imaging of lymphatic vessels was restricted to techniques linked to administration of specific dyes to the lymphatic system. The dyes used at present, e.g. Evans blue or trypan blue are much better tolerated than those used in the past [14]. The dyes and rhodamine dextran are routinely applied in studies on animals [18]. Until now, immunohistochemical identification of lymphatic vessels was possible due to comparison of expression of endothelial markers with those of basement membrane. For example, CD31, the expression of which is observed in blood and lymphatic vessels was used in combination with markers of basement membrane and collagen type IV [19]. The vessels which were labelled with CD31-specific antibodies but without expression of basement membrane proteins and containing no erythrocytes in their lumen, were taken as representing lymphatic vessels [20]. Use of a specific marker of blood vessels, PAL-E, in combination with PECAM-1 (platelet/endothelial cell adhesion molecule-1), was also helpful in identification of lymphatic vessels [21].

It was not until the recent few years that more numerous reports on lymphangiogenesis appeared. This was linked to distinguishing specific proteins which manifest expression in lymphatic vessels and to discovery of factors which control and stimulate growth of lymphatic vessels [22].

The recently discovered receptor for hyaluronic acid, located in endothelium of lymphatic vessels, LYVE-1 (lymphatic endothelium hyaluronate receptor) represents an important marker of lymphatic vessels [23] (Fig. 1). LYVE-1 plays a key role not only in metabolism, binding and transport of hyaluronic acid from tissues to lymphatic vessels but also in transplacement of leukocytes in lymphatic vessels and lymph nodes [24]. LYVE-1 in $41 \%$ manifests a structure homologous to CD44 molecule, the expression of which is accompanied by an elevated metastatic potential of tumour cells [25]. Expression of LYVE-1 is observed also in hepatic sinuses and in splenic endothelium [24,26,27]. Until now, LYVE-1 has not been detected in any non-sinusoidal vascular endothelium except of blood vessels in lungs [28] and in lymph nodes [27]. Expression of LYVE-1 in lymph nodes is not restricted to lymphatic vessels and sinuses but it is also present in high endothelium venules (HEV) [27]. Cells of the endothelium exhibit a cuboid or cylindrical shape, they are high and typically protrude to the lumen of the vessel. In this respect they are basically different from endothelial cells in other blood vessels $[29,30]$. Moreover, HEV represent the site in which lymphocytes emigrate from peripheral blood [31].

Another marker of lymphatic vessels involves the human transcription factor, Prox-1. It was identified for the first time in 1999 as a molecule which undergoes expression in cells of lymphatic endothelium. Prox-1 is involved in growth and elongation of lymphatic capillaries [32].

Another protein which is expressed in a mature lymphatic endothelium is neutropilin 2 (NRP2). The protein is a receptor which binds VEGF-C (vascular endothelial growth factor) and also plays role of a coreceptor for VEGFR-3 (the receptor for VEGF) [33].

An additional protein which is typical for lymphatic endothelium involves podoplanin, belonging 

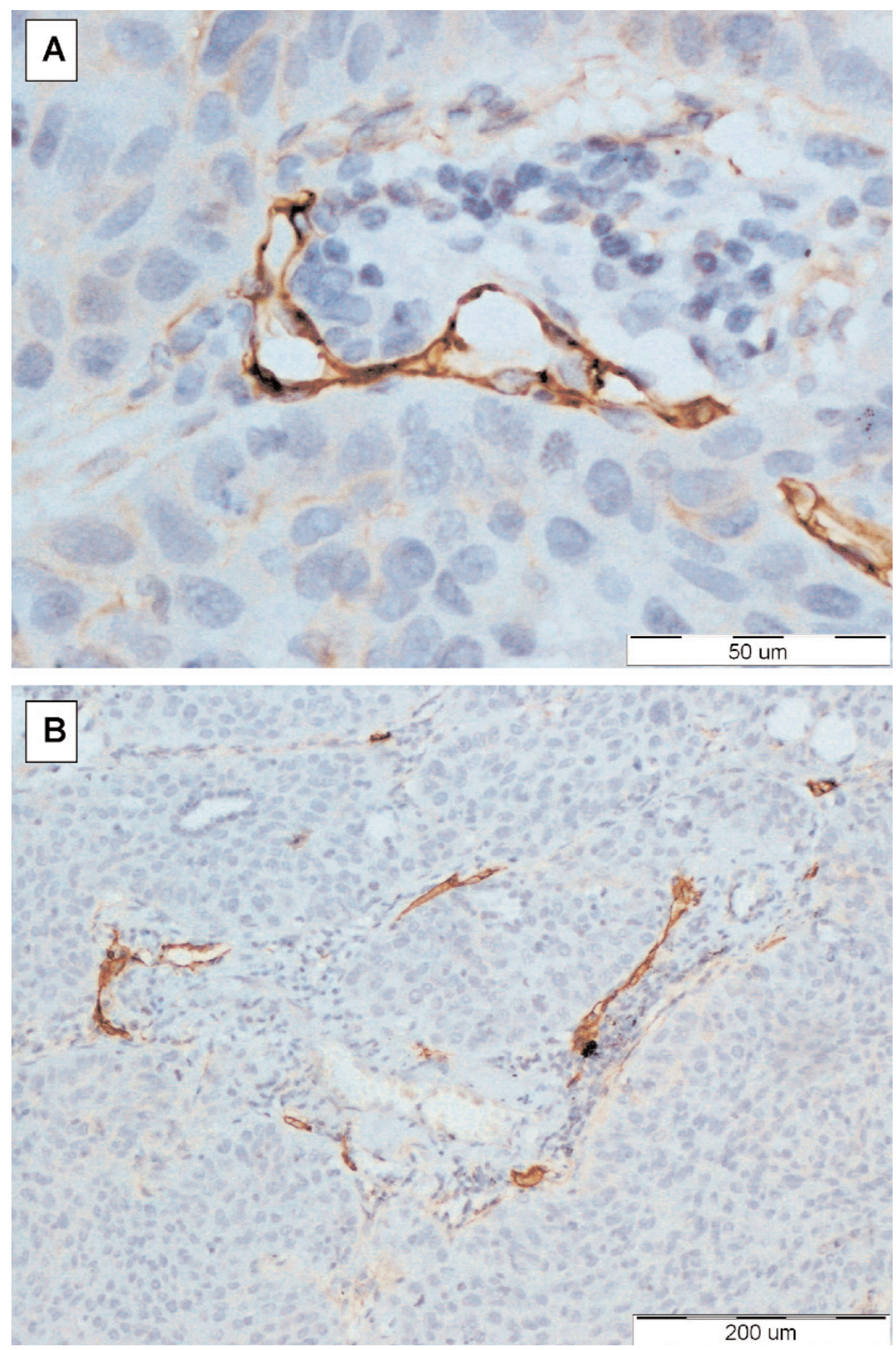

Fig. 1. Expression of LYVE-1 (brown) in lymphatic vessels in non-small cell lung cancer. A - magnification $\times 400$; B - magnification $\times 100$.

to the group of mucoproteins, the expression of which in endothelial cells of lymphatic vessels is controlled by Prox-1 [34]. Recently conducted studies indicate that podoplamin may participate in adhesion and migration of lymphatic endothelium cells [35].
Characterization of the proteins resulted not only in a design of novel techniques of identifying lymphatic vessels but permitted also to suggest a new approach to a lymphangiogenesis-modifying therapy of diseases associated with an abnormal structure and/or function 
of lymphatic vessels [36]. Currently, a great challenge is linked to definition of the detailed role played by LYVE-1 and other proteins engaged in immunological function of lymphoid system and recognition of the ways in which tumour cells take advantage of lymphatic vessels for development of metastases and, thus, for progression of the tumour.

\section{Factors which stimulate lymphangiogenesis}

Vascular endothelial growth factors (VEGF) are glycoproteins, the family of which encompasses VEGF-A, VEGF-B, VEGF-C, VEGF -D, VEGF- E and Placenta Growth Factor (PIGF) [37].

The vascular endothelial growth factor (VEGF), earlier termed the vascular permeability factor (VPF), capable of increasing vascular permeability, was discovered in 1989 and plays an important role in control of the of angiogenesis process $[38,39]$. All proteins of the VEGF family stimulate cell-mediated immunity by binding to their receptors (VEGFR), located on the surface of endothelial cells. VEGFR receptors consist each of the extracellular, transmembrane and intracellular domain, the latter manifesting activity of tyrosine kinase [40].

VEGF-A encompasses 10 isoforms: VEGF- $A_{121}$, VEGF-A $_{138}$, VEGF-A 145, VEGF-A 148, VEGF-A ${ }_{162}$, VEGF-A $_{162 b}$, VEGF-A 165, VEGF-A 183, VEGF-A 189 and VEGF- $\mathrm{A}_{206}$. It is synthesized by various cell types, including mast cells, smooth muscle cells of vascular walls, macrophages, fibroblasts, neoplastic cells, cells of endothelium, monocytes, keratinocytes, eosinophiles and lymphocytes T [41]. VEGF-A plays a key role in the process of angiogenesis [42].

VEGF-B in $88 \%$ resembles the structure of VEGF$\mathrm{A}$ and also participates in processes of angiogenesis and neurogenesis [43-46].

VEGF-C is indispensable in embryonal development in the process of formation of lymphatic vessels [47]. It manifests a mitogenic and a protective role related to both lymphatic and blood vessels [48]. A pronounced expression of VEGF-C is noted in the heart, placenta, muscles, ovaries, intestines and in certain tumours [49]. The factor is responsible also for increases in permeability and diameter of lymphatic vessels [50]. It seems to be of key importance in the process of lymphangiogenesis [51].

VEGF-D is expressed in the lungs, skin, heart, skeletal muscles, alimentary tract and in certain tumours [52]. It stimulates growth and migration of lymphatic endothelium cells [53]. Similarly to VEGF-C, VEGF-D participates also in the process of lymphangiogenesis [54].

VEGF-E, which till now has been located only in orf viruses, may be involved in the process of angiogenesis [55]. The orf virus, termed also the virus of ovine small- pox, belongs to the family of Poxviridae. The family encompasses large and structurally complex DNA viruses with a strong immunogenic potential.

The placenta growth factor (PIGF) fulfils an important role in both physiological and pathological angiogenesis. Similarly to VEGF it stimulates proliferation of endothelial cells. Originally, the factor was isolated for the first time from human placenta but later it was identified also in various tumours [56].

VEGFR-3 (Vascular Endothelial Growth Factor Receptor 3) proved to be the first specific receptor protein detected in lymphatic endothelium [57]. It fulfils the role of a receptor for lymphatic vessel endothelial growth factors VEGF-C and VEGF-D, belonging to the IIIrd class of tyrosine receptors [53]. Activation of VEGFR-3 by its ligands, VEGF-C and VEGF-D as well as the subsequent activation of its intracellular domain of tyrosine kinase stimulates proliferation of lymphatic endothelial cells [8]. Activity of lymphangiogenesis taking advantage of the VEGFR-3/VEGFC/VEGF-D axis was demonstrated in numerous investigations both in vivo and in vitro [58]. On the other hand, VEGFR-1 and VEGFR-2 receptors are characteristic for endothelium of blood vessels. The VEGFR2 receptor serves as the most important transmitter of signals in the process of angiogenesis but its presence was demonstrated also in lymphatic cisternae and lymphatic capillaries in the course of lymphangiogenesis [18]. The interaction between endothelial growth factors and VEGFR receptors have been presented by several authors $[11,59,60]$. In our review we would like to suggest a simple scheme of the above described relationships (Fig. 2).

\section{Lymphangiogenesis in malignant tumours}

The high mortality associated with neoplastic diseases used to ascribed, first of all, to spread in the body of neoplastic cells in the form of metastases. Clinical and anatomopathological reports confirm that metastases by lymphatic vessels to sentinel (regional) lymph nodes represent the early manifestation of the disease spread in cases of many solid tumours in humans [61].

Presence of neoplastic cells in the draining lymph nodes represents a significant index in evaluation of tumour advancement and in selection of the appropriate surgery, chemotherapy and radiotherapy. As mentioned earlier, the discovery of lymphatic vessel markers promoted development of studies using experimental models which allow for isolation of lymphatic endothelial cells. In recent years, increasing numbers of reports have appeared related to the role of lymphangiogenesis in mammary cancer, cancers of pancreas, prostate, stomach, in melanoma and pulmonary tumours as well as to growth factors specific for the processes [62-67]. 


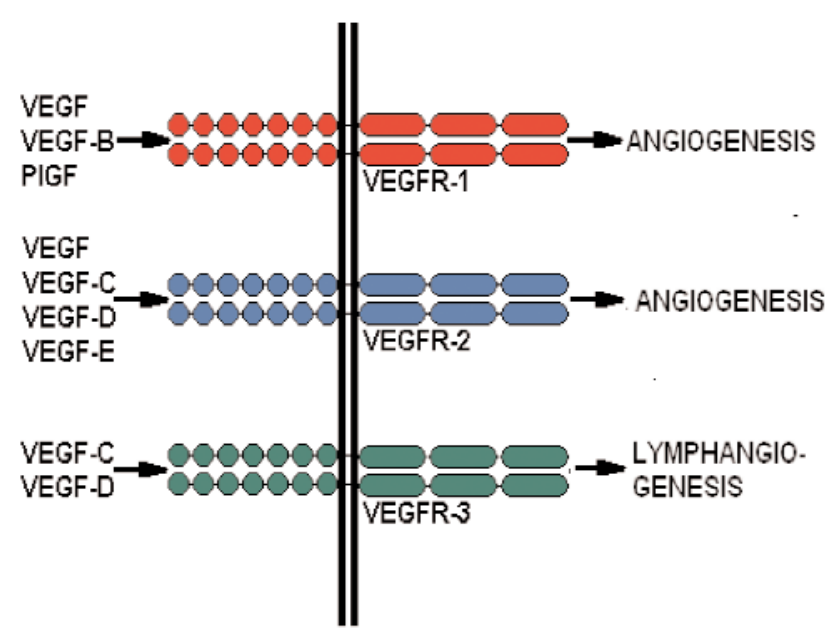

Fig. 2. Interactions between vasculoendothelial growth factors and their VEGFR receptors (60) in modification of our own. VEGF (VEGF-A) binds to VEGFR-1 and VEGFR-2. PIGF and VEGF-B are specific ligands for VEGFR-1. VEGF-E activates VEGFR-2. VEGF-C and VEGF-D interact with VEGFR-2 and VEGFR-3.

Dissemination of neoplastic cells from the primary tumour may take place by invasion of the local tissues, by the networks of lymphatic and blood vessels and by dissemination within body cavities. The most common method involves development of metastases due to spread of neoplastic cells with the circulating blood and within lymphatic vessels [67]. Schematic presentation of spread mechanisms and development of metastases from the tumour cells is shown in Fig. 3.

In 2005, He et al. [68] investigated the manner in which neoplastic cells enter lymphatic vessels and may form neoplastic metastases. The current concepts related to the mechanisms of metastasis formation are illustrated in Fig. 4A (in our own modification of the concept given in [67]). The neoplastic cells and macrophages which surround the tumour release VEGF-C and VEGF-D [8]. Activation of VEGFR-3 receptor by its ligands, VEGF-C and VEGF-D, leads to activation of its intracellular domain of tyrosine kinase, which stimulates proliferation and migration of lymphatic endothelial cells [69]. The mechanisms which promote the invasion of lymphatic vessels by neoplastic cells may include intra- and perineoplastic lymphangiogenesis as well as interactions between neoplastic cells and lymphatic endothelial cells (LEC). They result in an increase in penetrability of lymphatic vessels, altered adhesive properties of vascular endothelial cells, in this way facilitating development of metastases in lymph nodes [70].

The potential for inhibition of metastatic colonization of lymph nodes by tumour cells by inhibition of the VEGF-C/VEGF-D/ VEGFR-3 axis is shown in Fig. 4B. However, investigations of He et al. [68] demonstrated that inhibition of the VEGF-C/VEGF-D/

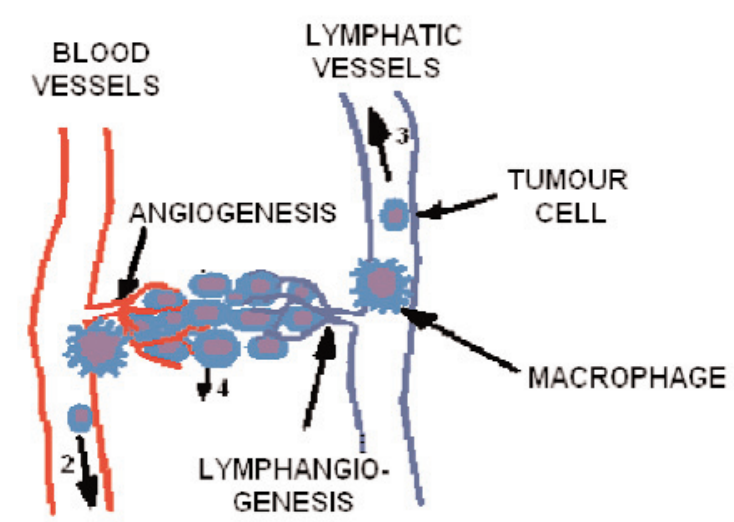

Fig. 3. Development of metastases (1) in continuity, in surrounding tissues, (2) by blood vessels, (3) by lymphatic vessels, (4) dissemination in body cavities (60, in modification of our own).

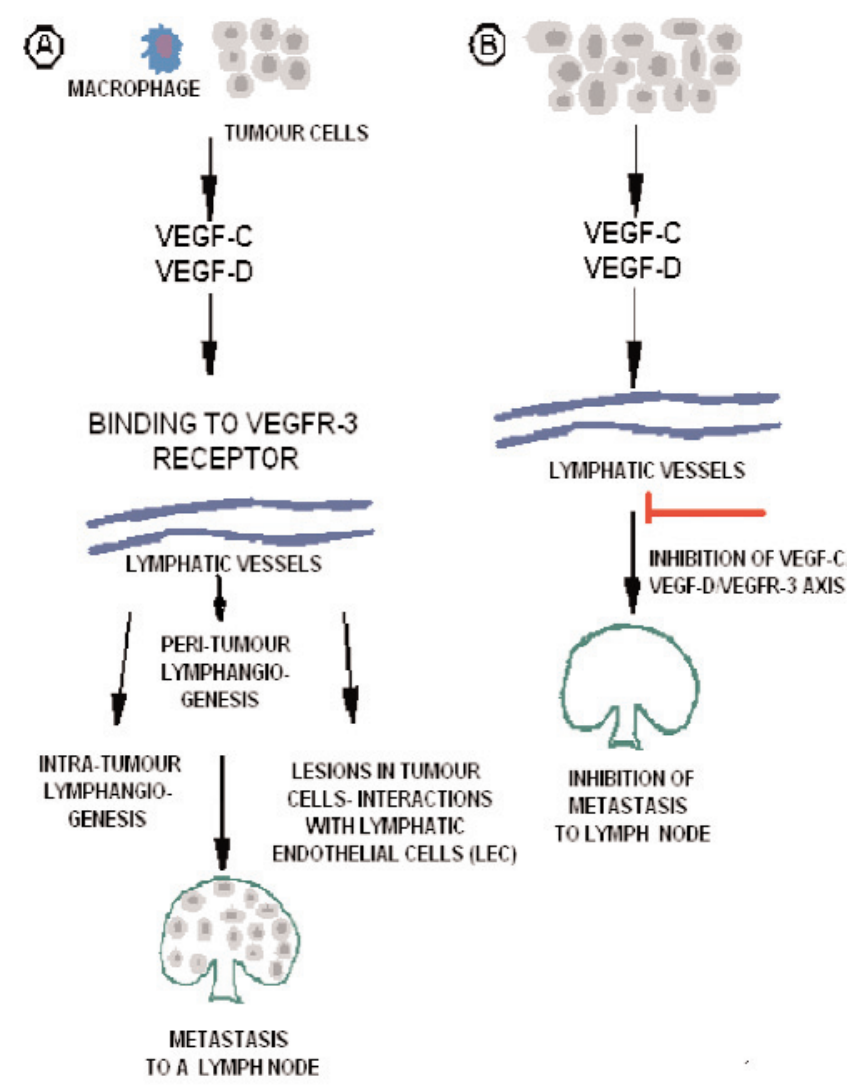

Fig. 4. A. Current concepts related to mechanisms in which neoplastic metastases develop using VEGFR-3/VEGF-C/VEGF-D axis. B. Potential for inhibition of metastases to lymph nodes by blocking VEGFR-3/VEGF-C/VEGF-D axis (67; in modification of our own).

VEGFR-3 axis was not sufficient to block development of metastases and the tumour cells still were present in the lymph nodes. Therefore, it seemed purposeful to inhibit lymphangiogenesis in parallel to blocking of the direct invasion of neoplastic cells from 
the primary tumour [68]. Activation of the VEGFC/VEGF-D/VEGFR-3 axis increases motility and invasiveness of neoplastic cells and in this way promotes development of neoplastic metastases in several types of tumours, e.g., in lung adenocarcinoma, cancers of mammary gland, neck, prostate and large intestine [71]. Most of the reports documented positive correlation noted between overexpression of VEGF-C and VEGF-D on one hand and invasion of lymphatic vessels, involvement of lymph nodes, development of distant metastases and an unfavourable clinical course on the other in various histological types of malignant tumours [72].

Also other lymphangiogenesis-stimulating factors were described as factors which negatively affect the course and prognosis in neoplastic diseases [65].

Cyclooxygenase 2 (COX-2) and its overexpression are associated with an unfavourable course of gastric as well as pulmonary adenocarcinoma and, in parallel, they stimulate lymphangiogenesis [73,74]. Also VEGF-C and COX-2 have been found to exhibit positive correlation with density of lymphatic microvessels and the extent of invasion of lymphatic vessels by neoplastic cells in gastric cancer [75].

Fibroblast growth factor 2 (FGF-2) induces both angiogenesis and lymphangiogenesis through the control of VEGF-C and VEGF-D expression, which has been confirmed in animal experiments [76].

Angiopoetin-1 and insulin-like growth factor (IGF) represent mediators in the action of VEGF-C/VEGFD/VEFGR-3 axis [77,78].

Even if VEFG-A plays a significant role in angiogenesis, some investigations point also to effects of VEGF-A on induction of lymphangiogenesis directly through activation of VEGFR-2 and indirectly, inducing an augmented secretion of VEGF-C and VEGF-D by macrophages in vicinity of neoplastic cells [79]. In view of the investigations it seems that treatment of tumours might yield improved effects upon parallel administration of VEGF-A/VEGFR-2 mechanism blockers, e.g. Avastin, and inhibitors of VEGFR-3 /VEGF-C/VEGF-D/ axis [57]. Recent studies on animal models, related to fibrosarcoma have demonstrated that platelet-derived growth factor (PDGF) induced neoplastic lymphangiogenesis and is responsible for development of intraneoplastic lymphatic vessels, which augments their potential to form intraneoplastic lymphatic vessels, thus stimulating their potential to develop metastases in lymph nodes [80]. In view of the above, extensive hope is linked to application of antagonists of platelet-derived growth factor, such as, e.g., Gleevec in treatment of neoplastic diseases [75].

Other new studies have shown that hepatocyte growth factor (HGF) participates in lymphangiogenesis by stimulating growth of peritumoral lymphatics (PTLs), due to activation of VEGFR-3 receptor [81].
Other currently conducted investigations also have demonstrated that nitrogen oxide (NO) is produced and released by cells of lymphatic endothelium and, probably due to its effect on penetrability of lymphatic endothelium, leads to stimulation of one of the isoforms of nitrogen oxide synthase (iNOS). In turn, activity of nitrogen oxide synthase in a tumour has been found to manifest positive correlation with expression of VEGF-C and VEGF-D [82].

\section{Lymphangiogenesis in lung cancer}

Lung cancer represent one of the most frequent causes of death due to neoplasia. Despite the progress in diagnosis and treatment prognosis in patients with lung cancer remains poor. Among many prognostic indices in non-small cell lung cancer presence of metastases to lymph nodes remains to be of principal prognostic significance [83]. Also, few data are available on mediating mechanisms in development of metastases to lymph nodes in lung cancer. The role of lymphangiogenesis in development of the tumour type continues to be studied. Studies on animal models related to breast and renal carcinomas unequivocally indicate that an increase in the level of VEGF-C or VEGF-D induces lymphangiogenesis [51,84]. Studies from the recent few years have indicated a positive correlation between expressions of VEGF-C/D and VEGFR-3 in non-small cell lung cancer [85].

In lung adenocarcinoma increase in the level of mRNA for VEGF-C is indicative of metastases to lymph nodes [86]. Moreover, a significantly longer survival has been shown in patients with non-small cell lun cancer manifesting low expression of VEGF$\mathrm{C}$ as compared to patients with high expression of VEGF-C [87].

In 2008 Kawai et al. [1] in patients with non-small cell lung cancer detected significant role of VEGF-A 121 isoform in promotion of lymphangiogenesis in sentinel lymph nodes situated most closely to the tumour. In order to evaluate lymphangiogenesis in the lymph nodes antibodies specific for LYVE-1 were used. In such patients, the authors detected a significantly elevated levels of LYVE-1 and VEGF-A 121 in sentinel lymph nodes as compared to the other lymph nodes. In the patients VEGF-A 121 was found to promote proliferation of lymphatic endothelium cells [1]. Moreover, the same authors detected a significant positive correlation between expression of VEGF-C and VEGF-D on one hand and expression of LYVE-1 expression in sentinel lymph nodes with neoplastic metastases. The relationship was absent from sentinel lymph nodes free of metastases [1].

Also in the year of 2008 Kadota et al. [88] evaluated lymphatic vessel density (LVD) and extent of invasion to lymphatic vessels (LVI) using antibodies specific for 
D2-40 (an equivalent of podoplanin) in patients with various types of non-small cell lung cancer. In the same patients density of intra-tumour microvessels (MVD) was also evaluated. A significantly higher lymphatic vessel density (LVD) was detected in squamous cell carcinoma as compared to adenocarcinoma. Shorter survival of the patients was linked to high LVD and high MVD. Lymphangiogenesis was found to be correlated with a high LVD. The data indicated that LVD and MVD represent independent prognostic indices in patients with non-small cell lung cancer [88].

The till now obtained results allow to conclude that high expression of VEGF-C correlates with presence of metastases to lymph nodes and with poor prognosis in patients with non-small cell lung cancer. The poor prognosis in such patients is also linked to high density of lymphatic vessels and a high density of intratumour microvessels.

Extensive hopes are associated also with drugs which inhibit lymphangiogenesis. Hopefully, in future improved results of pulmonary carcinoma treatment will be obtained due to application of a combined chemotherapy, consisting of, i.a., inhibitors of epidermal growth factor receptors and inhibitors of VEGFR3 receptor [89].

\section{Inhibition of lymphangiogenesis}

VEGFR-3 for VEGF represented the first to be described specific receptor protein for lymphatic endothelium [57]. It plays role of a receptor for the lymphatic endothelium growth factors, VEGF-C and VEGF-D. Recent investigations on animal models unequivocally proved that an increased level of VEGF-C and/or VEGF-D promotes lymphangiogenesis in neoplastic diseases and a lymphatic vessel-mediated tumour spread to regional lymph nodes. The phenomenon may be inhibited by blocking VEGFR-3 receptor [92].

Application of a soluble form of the VEGFR-3 receptor in lung cancer reduces the amount of intratumour lymphatic vessels as well as decreases frequency of metastases to regional lymph nodes and to the lungs $[69,92,93]$. Inhibitors of VEGFR-2 receptor proved to function also as inhibitors of VEGFR-3 receptor, such as Surafenib (Nexavar, BAY 43-9006) [89] and Cediranib (Recentin, AZD2171) [90] studied on lung cancer, SU 11248, studied on renal carcinoma [94]. Cediranib, examined by Heckman in 2008, significantly inhibited both angiogenesis and lymphangiogenesis in experimental models with adenoviruses [91]. MAZ 51 inhibited growth of breast cancer in studies on animal models [95]. Promising results were also obtained in studies on a recombined protein received from adenoviruses, which affected expression of the soluble form of VEGFR-3 receptor. The protein helped to inhibit metastases to lymph nodes and to decrease the amount of peri-tumour lymphatic vessels in mice $[51,96]$. In studies on animals with tumours secreting recombined VEGF-D, in which monoclonal antibodies to VEGF-D were used, which blocked ligand binding to both VEGFR-2 and VEGFR-3, the inhibited processes involved angiogenesis, lymphangiogenesis and spread of lymphatic vessel-mediated tumour metastases $[84,97]$. Also in recent years clinical studies succeeded in inhibiting growth of metastatic colonic and anal cancer due to application of Avastin preparation (Bevacizumab), the monoclonal antibody specific for VEGF (an inhibitor of angiogenesis) [98]. Duration of an average survival till progression of the disease and mean survival of the patients were found to be extended in the patients.

\section{Summary}

The problems which remain to be clarified include molecular mechanisms of lymphangiogenesis, specificity of growth factors, markers of lymphatic vessels and numerous phenomena responsible for tumour growth and its spread by lymphatic vessels. Recognition of angiogenesis and lymphangiogenesis and design of drugs which effectively inhibit the processes may significantly improve results of therapy in neoplastic diseases.

In recent years, lymphangiogenesis in pulmonary cancer constitutes a topic of intense studies. In the non-small cell lung cancer expressions of VEGF-C/D and of VEGFR-3 have been found to be positively correlated with each other.

A significantly abbreviated survival is noted in lung cancer patients with high expression of VEGF-C. Investigations conducted in 2008 have demonstrated positive correlation between expression of VEGF-C and VEGF-D on one hand and expression of LYVE-1 in sentinel lymph nodes with metastases of neoplastic cells on the other in patients with non-small cell lung cancer [1]. Also, high density of lymphatic vessels and a high density of intra-tumour microvessels have been demonstrated to represent independent unfavourable prognostic indices in patients with non-small cell lung cancer [88].

\section{References}

[ 1] Kawai H, Minamiya Y, Ito M. et al. VEGF121 promotes lymphangiogenesis in the sentinel lymph nodes of non-small cell lung carcinoma patients. Lung Cancer. 2008;59:41-47.

[2] Ginsberg MS. Epidemiology of lung cancer. Semin. Roentgenol. 2005;40:83-89.

[3] Szczuka I, Roszkowski-Śliż K. Lung cancer in Poland in 1970-2004. Pneumonol Alergol Pol. 2008;76:19-28.

[4] Greenlee RT, Hill-Harmon MB, Murray T, Thun M. Cancer statistics. Cancer J Clin. 2001;51:15-36.

[5] Rennie J, Rusting R. Making headway against cancer. Sci Am. 1996;275:56-59. 
[ 6] Folkman J, Klagsburn M. Angiogenic factors. Science. 1987; 235:442-447.

[ 7] Weidner N, Semple JP, Welch WR, Folkman J. Tumor angiogenesis and metastasis - correlation in invasive breast carcinoma. NEJM. 1991;324,1-8.

[ 8] Stacker SA, Baldwin ME, Achen MG. The role of tumor lymphangiogenesis in metastatic spread. FASEB J. 2002;16,922934.

[ 9] Ryan TJ. Structure and function of lymphatics. J Invest Dermatol. 1989;93:18S-24S.

[10] Clarijs R, Ruiter DJ, de Waal R.M. Lymphangiogenesis in malignant tumours: does it occur? J Pathol. 2001;193:43-146.

[11] Jussila L, Alitalo K. Vascular Growth Factors and Lymphangiogenesis. Physiol Rev. 2002;82:673-700.

[12] Leak LV, Burke JF. Fine structure of the lymphatic capillary and the adjoining connective tissue area. Am J Anat. 1966; 118:785-809.

[13] Roitt I, Brostoff J, Male D. Immunology. Mosby International. London. 1998.

[14] Skobe M, Detmar M. Structure, function, and molecular control of the skin lymphatic system. J Invest Dermatol. 2000;5: 14-19.

[15] Witte MH, Bernas MJ, Martin CP, Witte CL. Lymphangiogenesis and lymphangiodysplasia: from molecular to clinical lymphology. Microsc Res Tech. 2001;55:122-145.

[16] Hosking B, Makinen T. Lymphatic vasculature: a molecular perspective. BioEssay. 2007;29:1192-1202.

[17] Wigle JT, Harvey N, Detmar M. et al. An essential role for Prox 1 in the induction of the lymphatic endothelial cell phenotype. EMBO J. 2002;21:1505-1513.

[18] Veikkola T, Jussila L, Makinen T. Signalling via vascular endothelial growth factor receptor-3 is sufficient for lymphangiogenesis in transgenic mice. EMBO J. 2001;20:1223-1231.

[19] Nerlich AG, Schleicher E. Identification of lymph and blood capillaries by immunohistochemical staining for various basement membrane components. Histochemistry. 1991;96: 449-453.

[20] Lymboussaki A, Partanen TA,Olofsson B. et al. Expression of the vascular endothelial growth factor $\mathrm{C}$ receptor VEGFR - in lymphatic endothelium of skin and in vascular tumors. $\mathrm{Am} \mathrm{J}$ Pathol. 1998;153:395-403.

[21] Jussila L, Valtola R, Partanen T. et al. Lymphatic endothelium and Kaposi's sarcoma spindle cells detected by antibodies against vascular endothelial growth factor receptor-3. Cancer Res. 1998;58:1599-1604.

[22] Sleeman J.P, Krishnan J, Kirkin V. et al. Markers for the lymphatic endothelium: In search of the holy grail? Microsc Res Tech. 2001;55:61-69.

[23] Jackson DG. New molecular markers for the study of tumour lymhangiogenesis. Anticancer Res. 2001;21:4279-4283.

[24] Jackson DG, Prevo R, Clasper S, Banerji S. LYVE-1, the lymphatic system and tumor lymphangiogenesis. Trends Immunol. 2001; 22:317-321.

[25] Prevo R, Banerji S, Ferguson D, Clasper S, Jackson D. Mouse LYVE-1 is an endocytic receptor for hyaluronate in lymphatic endothelium. J Biol Chem. 2001;276:19420-19430.

[26] Carreira CM, NasserSM, di Tomaso E. et al. LYVE-1 is not restricted to the lymph vessels: expression in normal liver blood sinusoid and down-regulation in human liver cancer and cirrhosis. Cancer Res. 2001;61:8079-8084.

[27] Wróbel T, Dzięgiel P, Mazur G. et al. LYVE-1 expression on high endothelial venules ( HEVs) of lymph nodes. Lymphology. 2005;38:107-110.

[28] Koukourakis MI, Giatromanolaki A, Sivridis E. et al. LYVE1 immunohistochemical assessment of lymphangiogenesis in endometrial and lung cancer. J Clin Pathol. 2005; 58:202206.
[29] Girard JP, Springer TA. High endothelial venules (HEVs): specialized endothelium for lymphocyte migration. Immunol Today. 1995;16: 449-457.

[30] Duijvestijn AM, Rep M, Hendriks HR. et al. Functional capacities of high endothelial venules appear not to be controlled by recirculating lymphocytes. Immunobiology. 1990; 180:295-307.

[31] Faveeuw C, Preece G, Ager A. Transendothelial migration of lymphocytes across high endothelial venules into lymph nodes is affected by metalloproteinases. Blood. 2001;98:688-695.

[32] Rodriguez-Niedenfuhr M, Papoutsi M, Crist B. et al. Prox1 is a marker of ectodermal placodes, endodermal compartments, lymphatic endothelium and lymphangioblasts. Anat Embryol (Berl). 2001;204:399-406

[33] Yuan L, Moyon D, Pardanaud L. et al. Abnormal lymphatic vessel development in neutropilin 2 mutant mice. Development. 2002;129;4797-806.

[34] Breiteneder-Geleff S, Soleiman A, Kowalski H. et al. Angiosarcomas express mixed endothelial phenotypes of blood and lymphatic capillaries: podoplanin as a specific marker for lymphatic endothelium. Am J Pathol. 1999;154; 385-394.

[35] Schacht V, Ramirez MI, Hong YK. et al. T1alpha/podoplanin deficiency disrupts normal lymphatic vasculature formation and causes lymphedema. EMBO J. 2003;22:3546-3556.

[36] Scavelli C, Weber E, Agliano M. et al. Lymphatics at the crossroads of angiogenesis and lymphangiogenesis. J Anat. 2004;204:433-449.

[37] Ferrara N, Henzel WJ. Pituitary follicular cells secrete a novel heparin- binding growth factor specific for vascular endothelial cells. Biochem Biophys Res Commun. 1989;161:851-858.

[38] Leung DW, Cachianes G, Kuang WJ.et al. Vascular endothelial growth factor is a secreted angiogenic mitogen. Science. 1989;246:1306-1309.

[39] Ferrara N. VEGF: an update on biological and therapeutic aspects. Curr Opin Biotechnol. 2000;11:617-624.

[40] Gruchlik A, Chodurek E, Domal- Kwiatkowska D. et al. VEGF-A - the target of anti-angiogenic tumour therapy (in Polish). Post Biol Kom. 2007;3:557-580.

[41] Nörje CJ, Mooney DJ, Polverini PJ. Vascular Endothelial Growth Factor (VEGF) - mediated angiogenesis is associated with enhanced endothelial cell survival and induction of Bcl-2 expression. Am J Pathol. 1999;154:375-384.

[42] Saaristo A, Karpanen T, AlitaloK. Mechanisms of angiogenesis and their use in the inhibition of tumor growth and metastasis. Oncogene. 2000;19:6122-6129.

[43] Olofsson K, Pajusola A, Kaipanen G. et al. Vascular endothelial growth factor B, a novel growth factor for endothelial cells. Proc Natl Acad Sci USA. 1996;93:2576-2581

[44] Silvestre JS, Tamarat R, Ebrahimian TG. et al. Vascular endothelial growth factor-B promotes in vivo angiogenesis. Circ Res. 2003;93:114-123.

[45] Sun Y, Jin K, Childs JT. et al. Increased severity of cerebral ischaemic injury in vascular endothelial growth factor-B (VegfB)-deficient mice. J Cereb Blood Flow Metab. 2004; 24:1146-1152.

[46] Sun Y, Jin K, Childs JT. et al. Vascular endothelial growth factor-B(VEGFB) stimulates neurogenesis: Evidence from knockout mice and growth factor administration. Develop Biol. 2006;289:329-335.

[47] Karkkainen MJ, Haiko P, Sainio K. et al. Vascular endothelial growth factor $\mathrm{C}$ is required for sprouting of first lymphatic vessels from embryonic veins. Nat Immunol. 2004;5:74-80.

[48] Barańska P, Jerczyńska H, Pawłowska Z. Vascular endothelium growth factor: structure and functions (in Polish). Post Biochem. 2005;51:2-21.

[49] Ishikawa M. Kitayama J, Kazama S. et al. Expression of vascular endothelial growth factor $\mathrm{C}$ and $\mathrm{D}$ (VEGF-C and 
VEGF-D) is an important riskfactor for lymphatic metastasis in undifferentiated early gastric carcinoma. Jpn J Clin Oncol. 2003;33:21-27.

[50] Yonemura Y, Endo Y, Fujita H. et al. Role of vascular endothelial growth factor $\mathrm{C}$ expression in the development of lymph node metastasis in gastric cancer. Clin Cancer Res. 1999;5:1823-1829.

[51] Karpanen T, Egeblad M, Karkkainen MJ, Kubo H, Ylä- Herttuala S, Jäättelä M. Vascular endothelial growth factor C promotes tumor lymphangiogenesis and intralymphatic tumor growth. Cancer Res. 2001;61:1786-1790.

[52] Baldwin ME, Catimel B, Nice EC. et al. The specificity of receptor binding by vascular endothelial growth factor-D is different in mouse and man. J Biol Chem. 2001;276:1916619171.

[53] Karkkainen M, Alitalo K. Lymphatic endothelial regulation, lymphoedema and lymph node metastasis. Semin Cell Dev Biol. 2002;13:9-18.

[54] Kopfstein L, Veikkola T, Djonov VG. et al. Distinct roles of vascular endothelial growth factor-D in lymphangiogenesis and metastasis. Am J Pathol. 2007;170:1348-1361.

[55] Scagliarini A, Pozzo F, Gallina L. et al. In vitro activity of VEGF-E produced by orf virus strains isolated from classical and severe persistent contagious ecthyma. Vet Microbiol. 2006;114:142-147.

[56] Zielonka TM. Angiogenesis. Part II. Factors modulating the process of new blood vessel development (in Polish). Alergia Astma Immunologia. 2004;9:25-31.

[57] Kaipainen A, Korhonen J, Mustonen T. et al. Expression of the fms-like tyrosine kinase 4 gene becomes restricted to lymphatic endothelium during development. Proc Natl Acad Sci U.S.A. 1995;92:3566-3570.

[58] Achen MG, Stacker SA. Tumor lymphangiogenesis and metastasic spread-new players begin to emerge. Int J Cancer. 2006;119:1755-1760.

[59] Waś H. Characteristics of antigens and endothelial growth factors in lymph vessels (in Polish). Postep Biochem. 2005;2:209-214.

[60] Waś H. Lymphangiogenesis: the younger sister of angiogenesis? How Achilles tendon was detected in tumours (in Polish). Wszechświat. 2005;106:7-9.

[61] He Y, Karpanen T, Alitalo K. Role of lymphangiogenic factors in tumor metastasis. Biochim Biophys Acta. 1654. 2004;3-12.

[62] Skobe M, Hawighorst T, Jackson DG. et al. Induction of tumor lymphangiogenesis by VEGF-C promotes breast cancer metastasis. Nature Med. 2001;7:192-198.

[63] Rubbia-Brandt L, Terris B, Giostra E. et al. Lymphatic vessel density and vascular endothelial growth factor-C expression correlate with malignant behavior in human pancreatic endocrine tumors. Clin Cancer Res. 2004;10:6919-6928.

[64] Wong SY, Haack H, Crowley D. et al. Tumor-secreted vascular endothelial growth factor-C is necessary fo prostate cancer lymphangiogenesis, but lymphangiogenesis is unnecessary for lymph node metastasis. Cancer Res. 2005;65:9789-9798.

[65] Da MX, Wu Z, Tian HW. Tumor lymhangiogenesis and Lymphangiogenic Growth Factors. Arch Med Res. 2008;39:365372.

[66] Harrell MI, Iritani BM, Ruddell A. Tumor-induced sentinel lymph node lymphangiogenesis and increased lymph flow precede melanoma metastasis. Am J Pathol. 2007;170:774-786.

[67] Sundar SS, Ganesan TS. Role of Lymphangiogenesis in Cancer. J Clin Oncology. 2007;27:4298-4307.

[68] He Y, Rajantie I, Pajusola K, et al. Vascular endothelial cell growth factor receptor 3-mediated activation of lymphatic endothelium is crucial for tumor cell entry and spread via lymphatic vessels. Cancer Res. 2005;65:4739-4746.
[69] Mäkinen T, Jussila L, Veikkola T. et al. Inhibition of lymphangiogenesis with resulting lymphoedema in transgenic mice expressing soluble VEGF receptor-3. Nature Medicine. 2001;7:199-205.

[70] Shields JD, Borsetti M, Rigby H. et al Lymphatic density and metastatic spread in human malignant melanoma. $\mathrm{Br} J$ Cancer. 2004;90:693-700.

[71] Su JL, Yang PC, Shih JY. et al. The VEGF-C/VEGFR-3 axis promotes invasion and metastasis of cancer cells. Cancer Cell. 2006;9:209-223.

[72] Kyzas PA, Geleff S,Batistatou A. et al. Evidence for lymphangiogenesis and its prognostic implications in head and neck squamous cell carcinoma. J Pathol. 2005;206:170-177.

[73] Chen CN, Sung CT, Lin MT. et al. Clinicopathologic association of cyclooxygenase 1 and cyclooxygenase 2 expression in gastric adenocarcinoma. Ann Surg. 2001;233:183-188.

[74] Su JL, Shih JY, Yen ML. et al. Cyclooxygenase-2 induces EP1- and HER-2/Neu-Dependent Vascular Endothelial Growth Factor-C up-regulation: A novel mechanism of lymphangiogenesis in lung adanocarcinoma. Cancer Res. 2004; 64:554-564.

[75] Da MX, Wu XT, Wang J. et al. Expression of cyclooxygenase -2 and vascular endothelial growth factor-c correlates with lymphangiogenesis and lymphatic invasion in human gastric cancer. Arch Med Res. 2008;39:92-99.

[76] Chang LK, Garcia-Cardena G, Farnebo F. et al. Dose dependent response of FGF-2 for lymphangiogenesis. Proc Natl Acad Sci USA. 2004;101:11658-11663.

[77] Tammela T, Saaristo A, Lohela M. et al. Angiopoetin-1 promotes lymphatic sprouting and hyperplasia. Blood. 2005;105: 4649-4656

[78] Bjorndahl M, Cao R, Nissen LJ. et al. Insulin-like growth factors 1 and 2 induce lymphangiogenesis in vivo. Proc Natl Acad Sci USA. 2005;102:15593-15598.

[79] Alitalo K, Tammela T, Petrova TV. Lymphangiogenesis in development and human diseases. Nature. 2005;438:946-953.

[80] Cao R, Bjorndahl MA, Religa P. et al. PDGF-BB induces intratumoral lymphangiogenesis and promotes lymphatic metastasis. Cancer Cell. 2004;6:333-345.

[81] Cao R, Bjorndahl MA, Gallego MI. et al. Hepatocyte growth factor is a lymphangiogenic factor with an indirect mechanism of action. Blood. 2006;107:3531-3536.

[82] Nakamura Y, Yosuoka H, Zuo H. et al. Nitric oxide in papillary thyroid carcinoma: induction of vascular endothelial growth factor $\mathrm{D}$ and correlation with lymph node metastasis. $J$ Clin Endocronol Metab. 2006;91:1582-1585.

[83] Sihoe AD, Yim AP. Lung cancer staging. J Surg Res. 2004; 117:92-106

[84] Stacker SA, Caesar C, Baldwin ME. et al. VEGF-D promotes the metastatic spread of tumor cells via the lymhatics. Nature Med. 2001;7:186-191.

[85] Kajita T., Ohta Y., Kimura K. et al. The expression of vascular endothelial growth factor $\mathrm{C}$ and its receptors in non-small cell lung cancer. Br J Cancer. 2001;85,255-260.

[86] Niki T, Iba S, Tokunou M, Yamada T, Matsuno Y, Hirohashi S. Expression of vascular endothelial growth factors A, B, Cand D and their relationships to lymph node status in lung adenocarcinoma. Clin Cancer Res. 2000;6:2431-2439.

[87] Ogawa E, Takenaka K, Yanagihara K. et al. Clinical significance of VEGF-C status in tumor cells and stromal macrophages in non-small cell lung cancer patients. $\mathrm{Br} J$ Cancer 2004;91:498-503.

[88] Kadota K, Huang C, Liu D. i wsp. The clinical significance of lymphangiogenesis and angiogenesis in non-small cell lung cancer patients. Eur J Cancer. 2008;44:1057-1067.

[89] Blumenschein G. Sorafenib in lung cancer: clinical developments and future directions. J Thorac Oncol 2008;3:124127. 
[90] Hanrahan EO, Heymach JV. Vascular endothelial growth factor receptor tyrosine kinase inhibitors vandetanib (ZD6474) and AZD 2171 in lung cancer. Clin Cancer Res. 2007;13: 4617-4622.

[91] Heckman CA, Holopainen T, Wirzenius M. et al. The tyrosine kinase inhibitor Cediranib blocks ligand-induced vascular endothelial growth factor receptor-3 activity and lymphangiogenesis. Cancer Res. 2008;68:4754-4762.

[92] He Y, Kozaki K, Karpanen T. et al. Suppression of tumor lymphangiogenesis and lymph node metastasis by bloking vascular endothelial growth factor receptor 3 signalin. $J$ Natl Cancer Inst. 2002;94:819-825.

[93] Krishnan J, Kirkin V, Steffen A. et al. Differential in vivo and in vitro expression of vascular endothelial growth factor VEGF-C and VEGF-D in tumors and its relationship to lymphatic metastasis in immunocompetent rats. Cancer Res. 2003;63:713-722.

[94] Motzer RJ, Michaelson MD, Redman BG. et al. Activity of SU 11248, a Multitargeted Inhibitor of Vascular Endothelial Growth Factor Receptor and Platelet-Derived Growth Factor Receptor in Patients with Metastatic Renal Cell Carcinoma. $J$ Clin Oncol. 2006;24:16-24.
[95] Kirkin V, Thiele W, Baumann P. et al. MAZ51, an indolinone that inhibits endothelial cell and tumor cell growth in vitro, suppresses tumor growth in vivo. Int $J$ Cancer. 2004;112: 986-993.

[96] Lin J, Lalani AS, Harding TC. et al. Inhibition of lymphogenous metastasis using adeno-associated virus-mediated gene transfer of a soluble VEGFR-3 decoy receptor. Cancer Res. 2005;65:6901-6909.

[97] Achen MG, Roufail S, Domagala T. et al. Monoclonal antibodies to vascular endothelial growth factor - D block interactions with both VEGF receptor-2 and VEGF receptor-3. Eur J Biochem. 2000;267:2505-2515.

[98] Kabbinavar F, Hurwitz HI, Fehrenbacher L. et al. Phase II, randomized trial comparing bevacizumab plus fluorouracil (FU)/leucovorin(LV) with FU/LV alone in patients with metastatic colorectal cancer. J Clin Oncol. 2003;21;60-65.

Submitted: 9 February, 2009 Accepted: 6 June, 2009 